\title{
QCD and Electroweak Physics at LHC
}

\author{
Klaus Rabbertz* \\ on behalf of the ATLAS and CMS Collaborations \\ Institut für Experimentelle Kernphysik, KIT — Karlsruher Institut für Technologie, Germany \\ E-mail: klaus.rabbertzecern.ch
}

First LHC data have been collected and collisions at a center-of-mass energy of $7 \mathrm{TeV}$ are anticipated for the next months. The commissioning of the detectors and the re-establishment of the Standard Model in the new energy regime will be the main tasks for the experimental collaborations in the year to come. This report summarizes the measurement plans and performance expectations of the ATLAS and CMS experiments for a selected number of QCD and electroweak analyses with an emphasis on the early data taking phase. Some longer term prospects are pointed out.

RADCOR 2009 - 9th International Symposium on Radiative Corrections (Applications of Quantum Field Theory to Phenomenology)

October 25-30 2009

Ascona, Switzerland

\footnotetext{
* Speaker.
} 


\section{Introduction}

In November 2009 the Large Hadron Collider (LHC) at CERN has been restarted and first collisions at the injection energy of $\sqrt{s}=900 \mathrm{GeV}$ have been registered. On the 13th of December the proton beams could even be accelerated up to $1.18 \mathrm{TeV}$ and achieved a record collision energy of $2.36 \mathrm{TeV}$ dethroning the Tevatron at Fermilab as the most powerful particle accelerator in the world. A new era in particle physics has just started and even higher center-of-mass energies up to $7 \mathrm{TeV}$ are anticipated for the next months.

Reaching, however, the high level of understanding of the complex detectors and the accelerator that is required in order to perform measurements as accurate as reported here [1] from the Tevatron experiments CDF and D0 will take time. The commissioning of the detectors and the re-establishment of the Standard Model in the new energy regime will therefore be the main tasks for the experimental collaborations in the year to come. This report summarizes the measurement plans and performance expectations of the ATLAS and CMS experiments for a selected number of QCD and electroweak analyses with an emphasis on this early data taking phase. Some longer term prospects are pointed out. Detailed descriptions of the LHC and the four main experiments can be found elsewhere $[2,3,4,5,6]$.

\section{Minimum Bias and Underlying Event}

Charged-particle multiplicity distributions versus pseudorapidity $\eta=-\ln \tan (\theta / 2$ ) (with $\theta$ being the polar angle) and transverse momentum $p_{T}$ have a long tradition in hadron-hadron collisions and have been measured e.g. in experiments at the ISR, SPS, Tevatron and RHIC colliders $[7,8,9,10]$. Since in terms of theory only models are available, the predictions for higher energies vary significantly even though they have been tuned to describe currently available data. The expectations by the ATLAS Collaboration [11] from PYTHIA [12] and PHOJET [13] for the average charged-particle density at central rapidity versus the center-of-mass energy is presented in Figure 1 left. An example of the reconstruction of the charged-particle density $d N_{c h} / d \eta$ using the ATLAS tracking for $|\eta|<2.5$ at $14 \mathrm{TeV}$ is given in Figure 1 right [11].

Corresponding expectations by CMS for $14 \mathrm{GeV}$ using full track reconstruction [14] or a pixel hit-counting technique [15], first developed by the PHOBOS experiment [16], are shown in Figure 2. Another method at $10 \mathrm{TeV}$ is described by CMS in [17].

Since only a few thousand events are required for these measurements they can be performed already early after the turn-on of a new accelerator. In fact, the very first measurement on LHC data, albeit at only $900 \mathrm{GeV}$ center-of-mass energy, has been published in the meantime by the ALICE collaboration [18]. Unsurprisingly, the new results are in line with previous measurements. Predicted differences between $p p$ and $p \bar{p}$ scattering at the order of some permille are well below the uncertainties and could not be observed. New publications from the LHC experiments including also the collision data at $2.36 \mathrm{GeV}$ center-of-mass energy should be expected soon. In [19] also further preliminary observations with the first LHC data have been reported. 

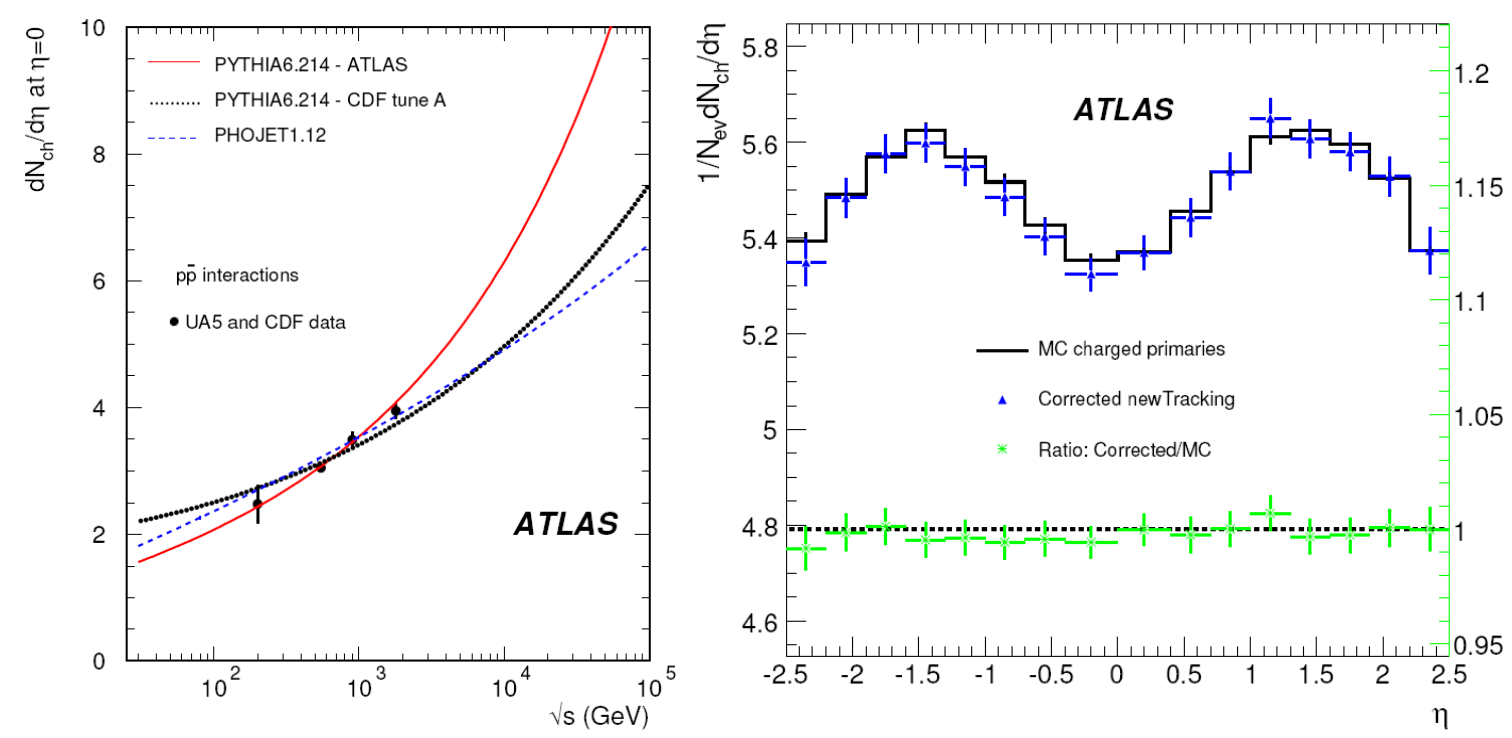

Figure 1: The average charged-particle density at central rapidity as a function of the center-of-mass energy is deduced from simulations with different tunes of the MC models PYTHIA and PHOJET on the left [11]. An example of the reconstruction of the charged-particle density using the ATLAS tracking for $|\eta|<2.5$ at $14 \mathrm{TeV}$ is given on the right [11].
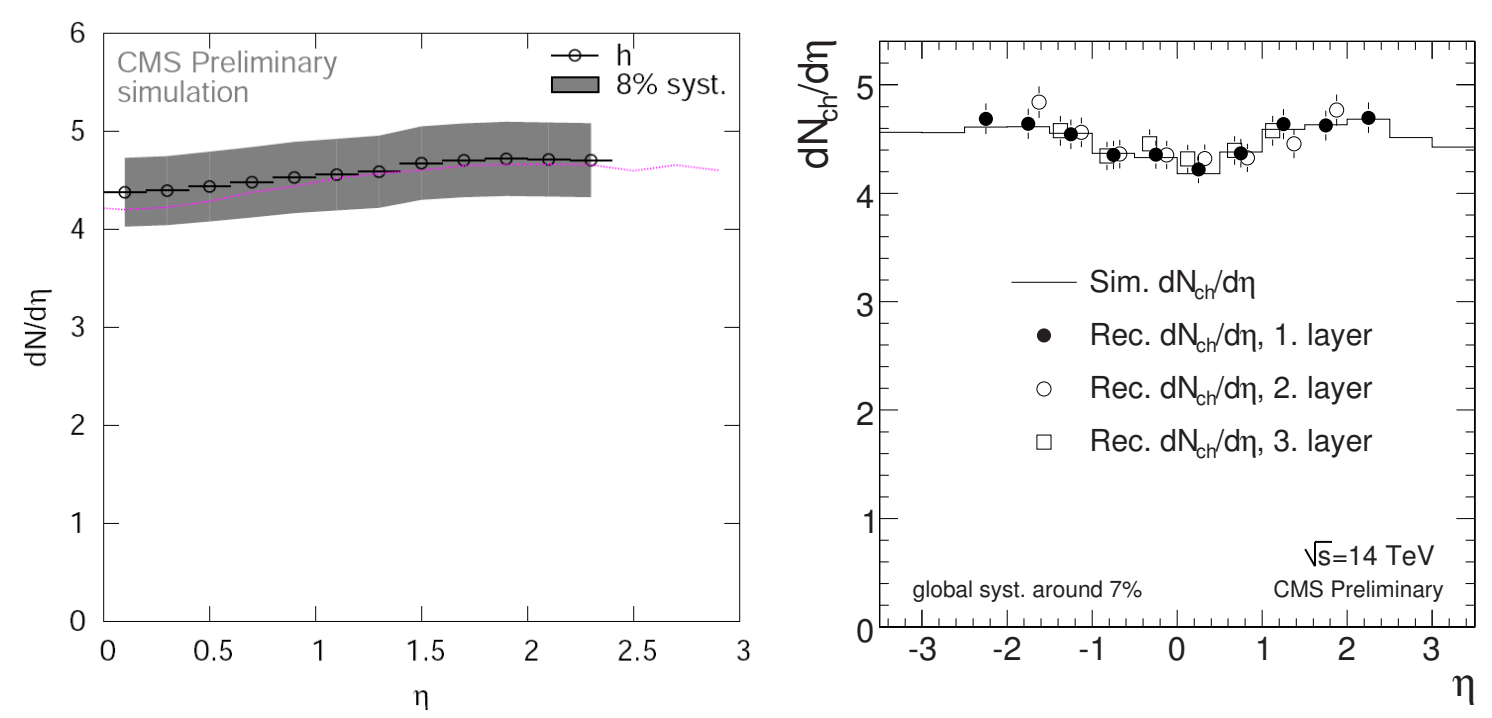

Figure 2: Pseudorapidity dependence of the charged-particle multiplicity at $14 \mathrm{TeV}$ by full track reconstruction [14] as well as by applying a pixel hit-counting method to PYTHIA events simulated for the CMS detector [15]. 
Another related topic exploits the fact that the transverse region of $60^{\circ}<|\Delta \phi|<120^{\circ}$ with respect to the leading jet in an event is most sensitive to the Underlying Event, i.e. every collision product not coming directly from the hard scatter [20,21]. Extrapolations of the UE contributions to events at LHC energies vary widely such that an early determination of its size and the tuning of the MC generators is an important start-up measurement.

Figure 3 presents the composition of the total charged-particle distribution in $\Delta \phi$ collected with Minimum Bias and jet triggers with different jet $p_{\mathrm{T}}$ thresholds on the left and the resulting $p_{\mathrm{T}}$ dependence of the charged-particle density in the transverse plane on the right [22] as reconstructed from CMS simulations with PYTHIA tune DWT. For comparison the MC predictions of PYTHIA with various tunes and from HERWIG without model for multiple parton interactions are shown as well. Already with the assumed $10 \mathrm{pb}^{-1}$ of integrated luminosity at $\sqrt{s}=14 \mathrm{TeV}$ it will be possible to differentiate between the extrapolations of some models to LHC energies. Note that in Figure 3 right tracks with a lower limit of $p_{\mathrm{T}}>500 \mathrm{MeV}$ have been chosen to further increase the sensitivity.
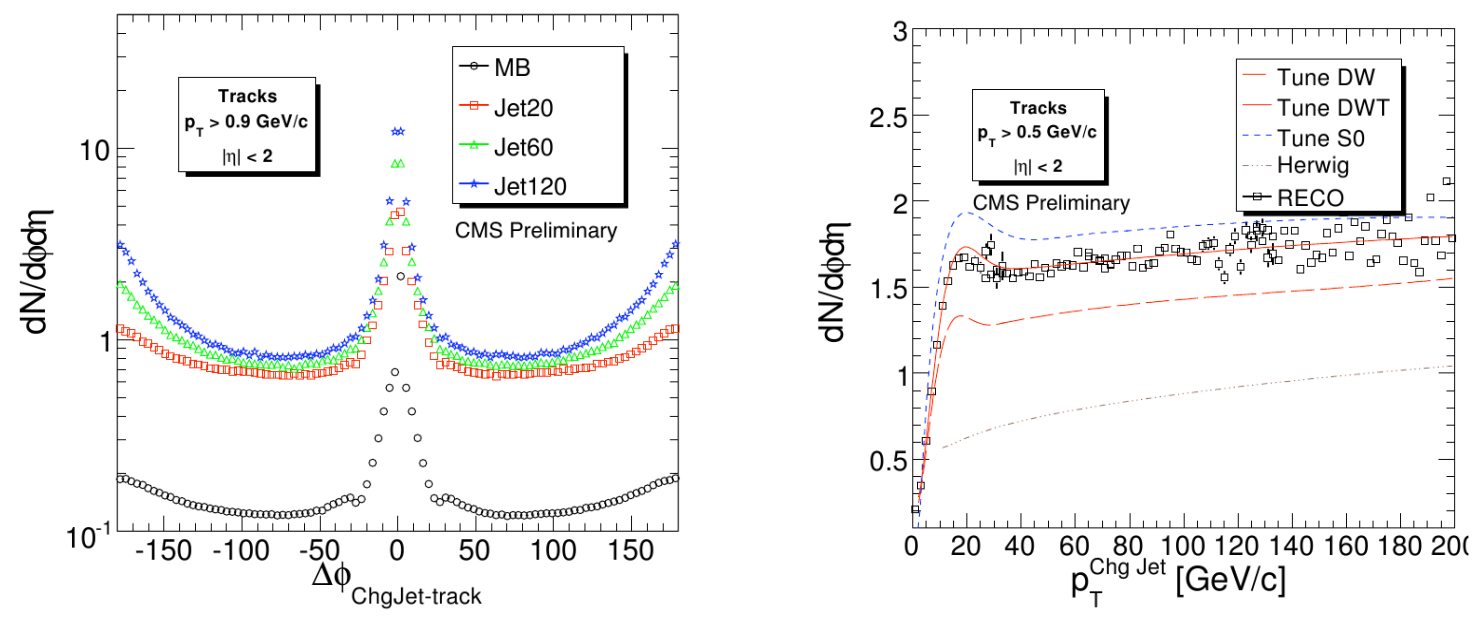

Figure 3: Composition of the total charged-particle distribution in $\Delta \phi$ for all trigger streams (left) and the resulting $p_{\mathrm{T}}$ dependence of the reconstructed charged-particle density in the transverse plane together with predictions of various PYTHIA tunes and from HERWIG assuming $10 \mathrm{pb}^{-1}$ of integrated luminosity at $\sqrt{s}=14 \mathrm{TeV}[22]$.

\section{Jet Measurements}

In order to establish a closer connection to the hard process which is described theoretically in terms of partons, i.e. quarks, anti-quarks and gluons, jet algorithms are employed. Although it is impossible to unambiguously assign bunches of observed hadrons to the originating partons, one can define a distance measure between objects and uniquely determine which of them are sufficiently close to each other to be considered to belong to the same jet or respectively to have a common origin.

In total six different jet algorithms with jet sizes $R$ (or $D$ ) ranging from 0.4 to 0.7 are in use by the ATLAS and CMS collaborations out of which two Iterative Cone algorithms (ICone-PR, IConeSM, see [23]) are not safe with respect to comparisons with theory calculations in perturbative 
QCD and therefore are not considered further. The remaining four are the Seedless Infrared-Safe Cone algorithm (SISCone) [24] and three algorithms of the sequential recombination type: The $k_{\mathrm{T}}$ [25, 26, 27], the Cambridge/Aachen [28] and the anti- $k_{\mathrm{T}}$ algorithm [23].

The standard jet measurement performed at all previous colliders so far is the differential inclusive jet production cross section. Unfortunately, it is affected by practically all the dominant experimental uncertainties due to the jet energy calibration (JEC), the luminosity determination, the jet energy resolution (JER), trigger efficiencies, and, less important, the spatial resolutions in azimuthal angle and pseudorapidity.

The reach in jet transverse momentum, however, is beyond any previous collider experiment $[29,30,31]$ already with $10 \mathrm{pb}^{-1}$ of integrated luminosity at a center-of-mass energy of $\sqrt{s}=10 \mathrm{TeV}$. Indications of new physics like from contact interactions would clearly be observable as demonstrated by CMS in [32]. Figure 4 compares the inclusive jet cross section in such a contact interaction scenario for a compositeness scale of $\Lambda^{+}=3 \mathrm{TeV}$ with the pure QCD prediction including estimates of all relevant experimental and theoretical uncertainties.
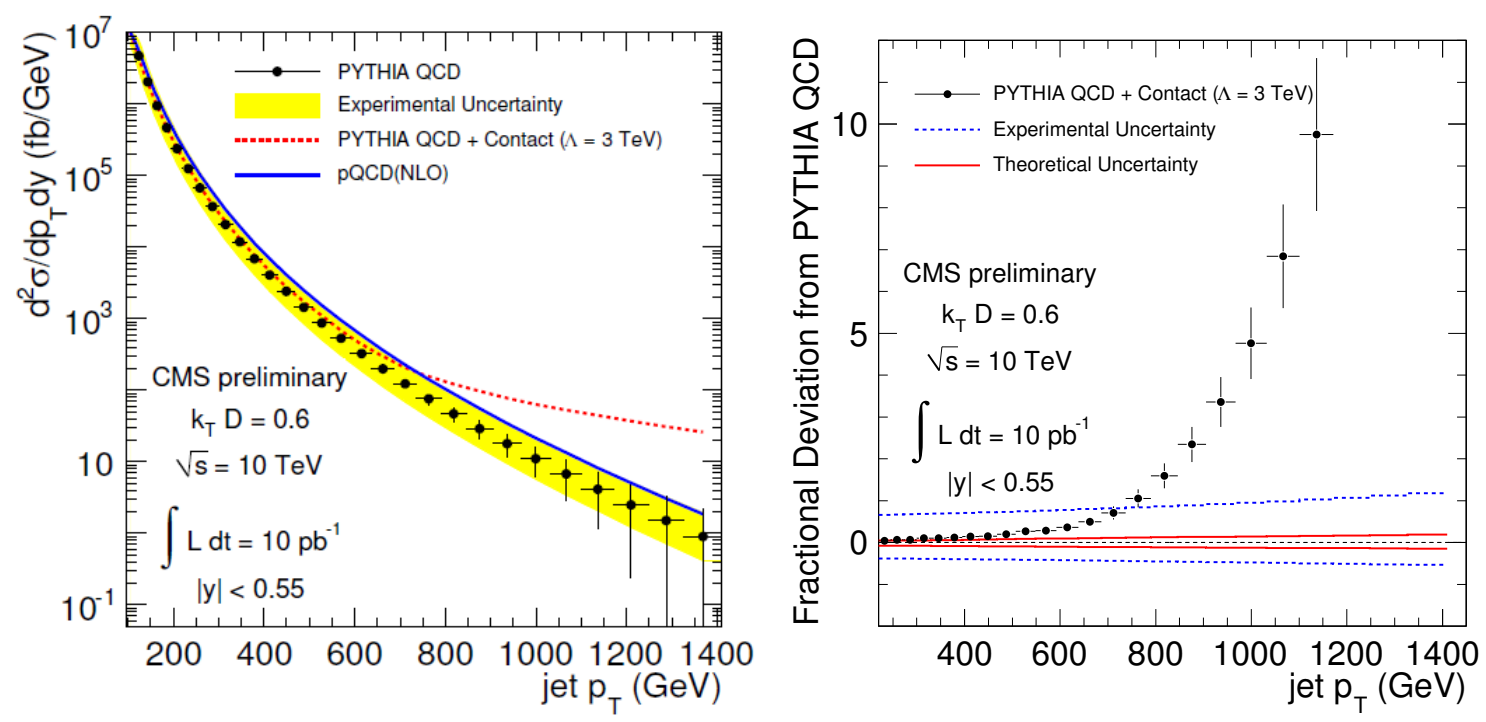

Figure 4: Measured inclusive jet spectrum (K factors times PYTHIA with CMS simulation) with experimental systematic uncertainty compared with theory (NLO times non-perturbative corrections) and PYTHIA QCD+3 TeV contact interaction term (left). Fractional difference of the QCD+contact interaction term and pure PYTHIA QCD is shown in comparison to the experimental and theoretical uncertainties (right).

Examples for jet observables less sensitive to experimental uncertainties are angle-related and/or normalized like dijet azimuthal decorrelations and event shapes or cross-section ratios like the dijet production ratio in pseudorapidity and 3-jet to all-jet ratios. Here, the luminosity uncertainty is eliminated and the uncertainty due to the JEC is largely reduced. Expectations for LHC with this type of analyses can be found for example in the references [33, 34, 35].

\section{Weak Boson Cross-Sections, $W$ Mass}

In contrast to the previously presented reactions the production rates for the weak bosons are orders of magnitudes smaller than for Minimum Bias or jet events (depending on $p_{\mathrm{T}}$ ). Nevertheless 
approximate rates of $10 / \mathrm{s}$ resp. $3 / \mathrm{s}$ for $W$ resp. $Z$ bosons and theoretical uncertainties smaller than $1 \%$ allow for precision measurements that stringently test the Standard Model in a new energy regime. Experimental uncertainties are well under control as long as the leptonic decay modes into muons and electrons are concerned. For selections of isolated leptons plus missing transverse momentum or unlike-sign lepton pairs within $\left|\eta_{l}\right|<2.5$ and for $p_{\mathrm{T}}>15-25 \mathrm{GeV}$ an accuracy of the $W$ and $Z$ production cross sections of about $5 \%$ for $W$ 's and 3\% for $Z$ 's is expected by ATLAS for an integrated luminosity of $50 \mathrm{pb}^{-1}$ at $14 \mathrm{TeV}$ [11]. With $1 \mathrm{fb}^{-1}$ of data irreducible uncertainties of about $1-2 \%$ are anticipated. Corresponding estimations by CMS can be found in [36, 37].

Improving on the $W$ mass is more difficult, especially considering the reduced uncertainty of $\Delta M_{W}=31 \mathrm{MeV}$ from $\mathrm{CDF}$ and D0 presented at this conference [1]. Compared to the PDG Review 2009 [38] with $\Delta M_{W}=40 \mathrm{MeV}$ from Tevatron and $25 \mathrm{MeV}$ as world combination this is a remarkable progress. On a longer timescale ATLAS predicts an achievable precision of $\mathscr{O}(<$ $10 \mathrm{MeV}$ ) for $10 \mathrm{fb}^{-1}$ of integrated luminosity at $14 \mathrm{TeV}$ provided radiative corrections are under control on the theory side [39, 40]. An example for a $W$ transverse mass distribution in the $W \rightarrow e v_{e}$ channel for $50 \mathrm{pb}^{-1}$ at $14 \mathrm{TeV}$ is displayed in Figure 5 left.
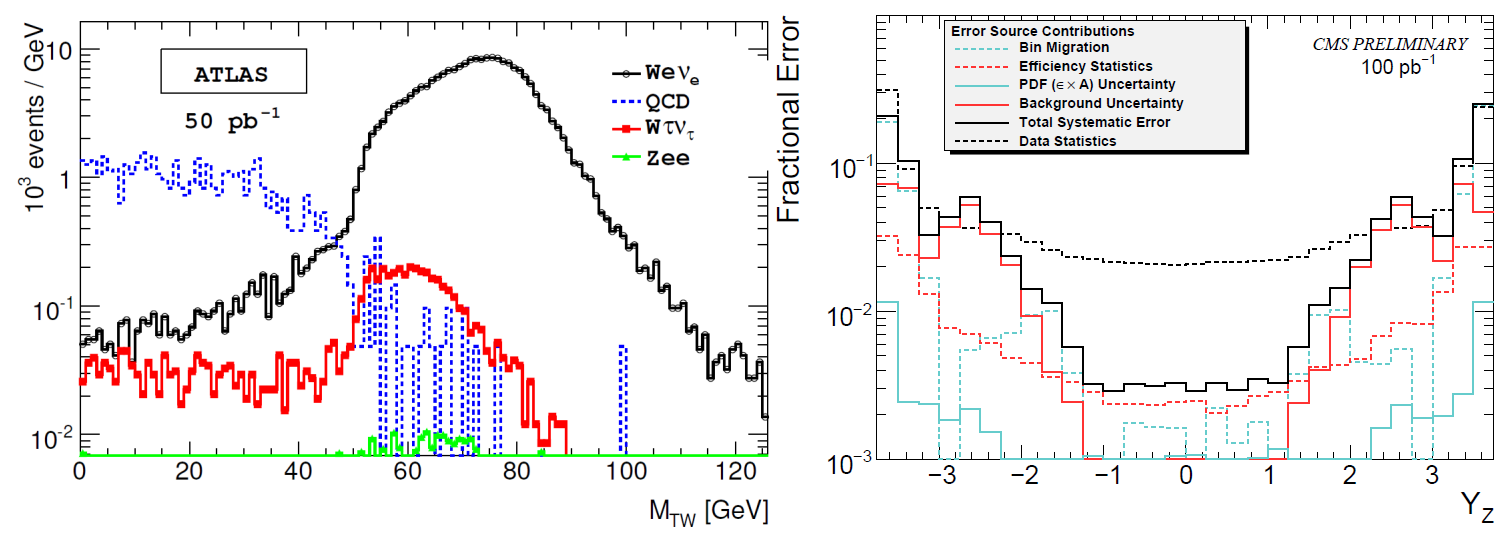

Figure 5: $W$ transverse mass distribution in the $W \rightarrow e v_{e}$ channel (left) [11] and fractional uncertainties of a $Z$ rapidity measurement (right) [41].

\section{Differential Weak Boson Measurements}

With somewhat more integrated luminosity the differential distributions of the weak bosons, in particular for the $Z$, can be exploited where the $Z$ transverse momentum provides even more constraints on QCD, especially on non-perturbative effects of initial parton emissions, while the rapidity distribution directly probes the parton density functions (PDFs) of the proton. Estimates on the fractional uncertainties for the $Z$ rapidity distribution from CMS [41] are displayed in Figure 5 right. Equally, the $W$ charge asymmetry shown in Figure 6 left can start constraining the PDFs with only $50 \mathrm{pb}^{-1}$ of data [42]. On a much longer timescale, once, about $100 \mathrm{fb}^{-1}$ of integrated luminosity at highest LHC energies have been accumulated, the weak mixing angle comes in reach for improving its accuracy. This has been studied by ATLAS in [11]. The lever arm for this is pictured in Figure 6 right. 

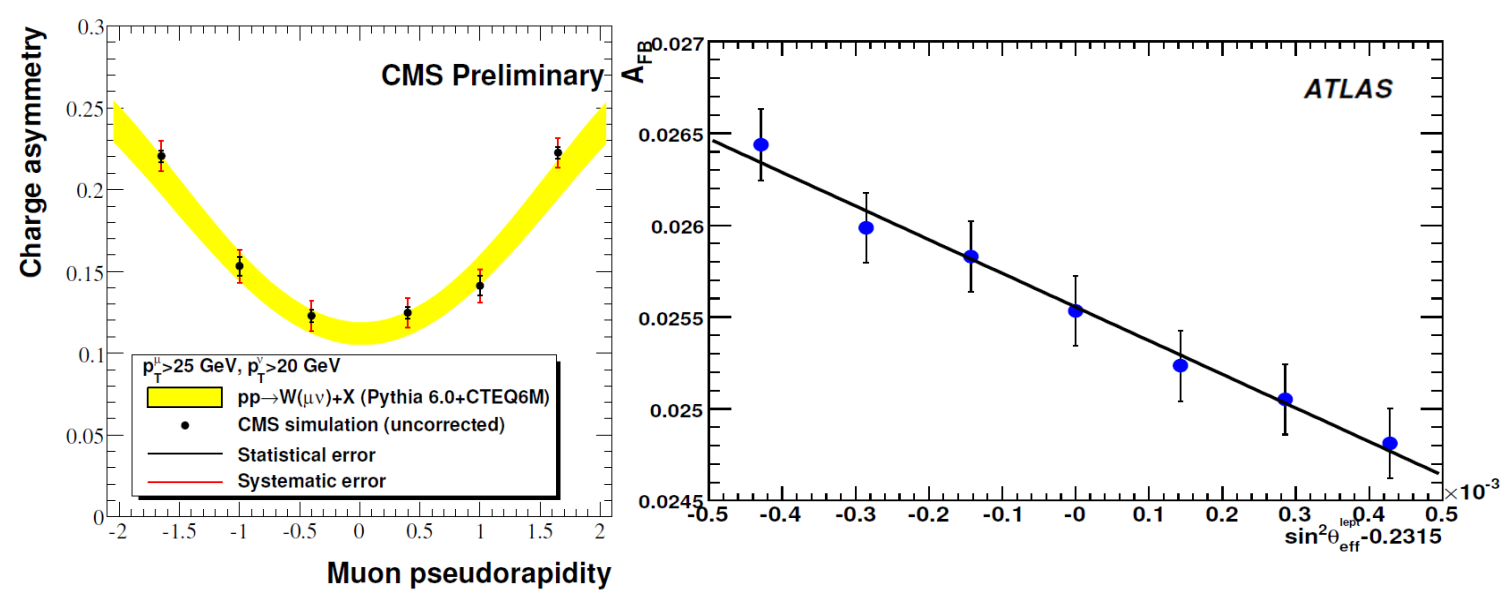

Figure 6: The reconstructed $W$ charge asymmetry including estimated statistical and systematic uncertainties for $100 \mathrm{pb}^{-1}$ of simulated luminosity at $10 \mathrm{TeV}$ from CMS (left) [42] and the forward backward asymmetry $A_{F B}$ versus the weak mixing angle $\sin ^{2} \theta_{\text {eff }}^{\text {lept }}$ at the $Z$ pole for $100 \mathrm{fb}^{-1}$ of integrated luminosity at $14 \mathrm{GeV}$ from ATLAS (right) [11].

\section{Boson plus Jet and Di-Boson Production}

Finally, the much higher center-of-mass energy at the LHC allows for more precise studies than ever before of multiple boson or of boson plus jet production. Figure 7 shows an estimate on the fractional uncertainties for the $Z+$ jet cross sections in the $Z \rightarrow e e$ channel (left) [43] as well as the $p_{\mathrm{T}}$ distribution of candidate lepton pairs for $W W$ di-boson events together with backgrounds (right) [11] both from ATLAS for $1 \mathrm{fb}^{-1}$ of integrated luminosity at $14 \mathrm{TeV}$. A study by CMS on $Z+$ jet production can be found in [44]. The CMS potential for measuring $W W$ production with $100 \mathrm{pb}^{-1}$ at $10 \mathrm{TeV}$ is reported in [45].
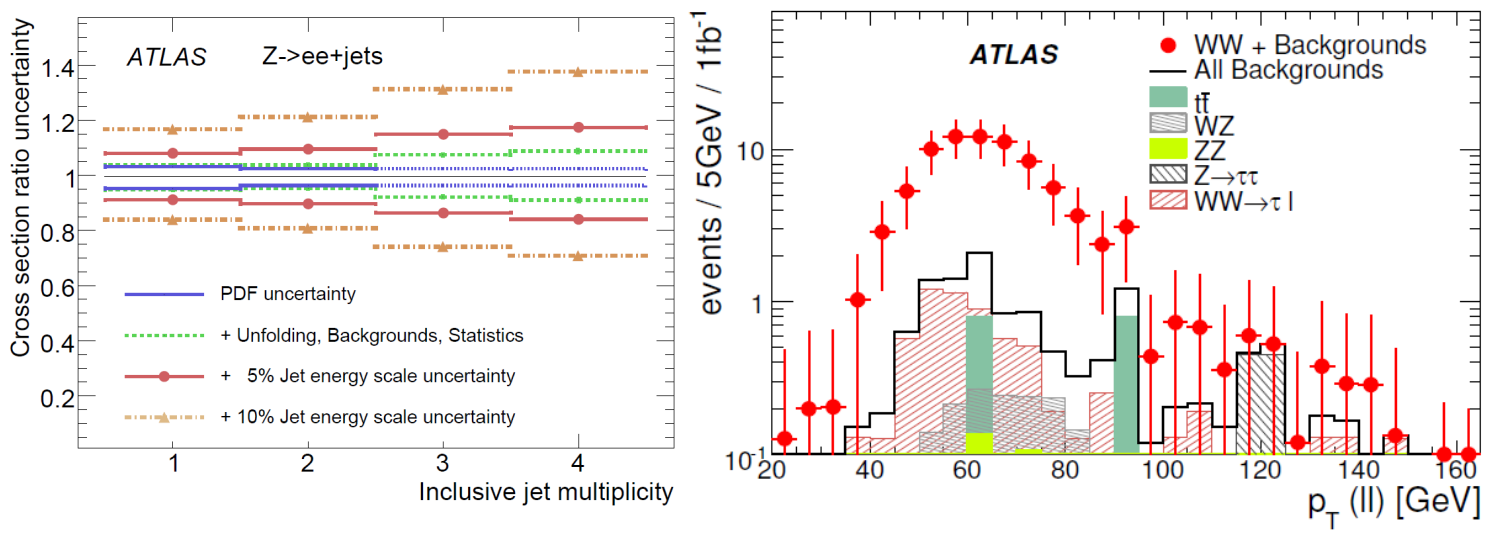

Figure 7: Relative uncertainties on a data-theory comparison of the $Z+$ jet multiplicity cross sections in the $Z \rightarrow e e$ channel (left) [43] and the $p_{\mathrm{T}}$ distribution of lepton pairs for simulated $W W$ candidate events (right) [11] both for $1 \mathrm{fb}^{-1}$ of integrated luminosity at $14 \mathrm{TeV}$. 


\section{Outlook}

The first LHC collision data have been registered up to a center-of-mass energy of $2.36 \mathrm{TeV}$ and the LHC experiments are in full swing of commissioning their detectors and publishing first physics results. Even higher energies will be reached in the very near future opening up a window to an unprecedented multitude of physics analyses involving multiple jet and boson production studies that were not possible before. The rich program of new physics measurements not only re-establishes the Standard Model but also sets the scene for searches for new phenomena. This year marks the beginning of a new era of particle physics.

\section{Acknowledgments}

I would like to thank the organizers for the kind invitation to participate in this conference.

\section{References}

[1] R. Wallny. these proceedings.

[2] L. Evans, (ed. ) and P. Bryant, (ed. ), LHC Machine, JINST 0803 (2008) S08001.

[3] ALICE Collaboration, The ALICE experiment at the CERN LHC, JINST 0803 (2008) S08002.

[4] ATLAS Collaboration, The ATLAS Experiment at the CERN Large Hadron Collider, JINST 0803 (2008) S08003.

[5] CMS Collaboration, The CMS experiment at the CERN LHC, JINST 0803 (2008) S08004.

[6] LHCb Collaboration, The LHCb Detector at the LHC, JINST 0803 (2008) S08005.

[7] Aachen-CERN-Heidelberg-Munich Collaboration, W. Thome et al., Charged Particle Multiplicity Distributions in p p Collisions at ISR Energies, Nucl. Phys. B129 (1977) 365.

[8] UA5 Collaboration, G. J. Alner et al., Scaling of Pseudorapidity Distributions at c.m. Energies Up to 0.9-TeV, Z. Phys. C33 (1986) 1-6.

[9] CDF Collaboration, F. Abe et al., Pseudorapidity distributions of charged particles produced in $\bar{p} p$ interactions at $\sqrt{s}=630 \mathrm{GeV}$ and $1800 \mathrm{GeV}$, Phys. Rev. D41 (1990) 2330.

[10] PHOBOS Collaboration, R. Nouicer et al., Pseudorapidity distributions of charged particles in $d+A u$ and $p+p$ collisions at $\sqrt{s_{\mathrm{NN}}}=200 \mathrm{GeV}$, J. Phys. G30 (2004) S1133, [nucl-ex $/ 0403033$ ].

[11] ATLAS Collaboration, G. Aad et al., Expected Performance of the ATLAS Experiment - Detector, Trigger and Physics, Tech. Rep. CERN-OPEN-2008-020. ISBN978-92-9083-321-5, CERN, Geneva, Sept., 2008.

[12] T. Sjostrand, S. Mrenna, and P. Skands, PYTHIA 6.4 physics and manual, JHEP 05 (2006) 026, [hep-ph/0603175].

[13] F. W. Bopp, R. Engel, and J. Ranft, Rapidity gaps and the PHOJET Monte Carlo, hep-ph/9803437.

[14] CMS Collaboration, Measurement of charged hadron spectra in proton-proton collisions at $\sqrt{s}=14 \mathrm{TeV}$, CMS Physics Analysis Summary CMS-PAS-QCD-07-001 (2007). 
[15] CMS Collaboration, Pseudorapidity distributions of charged hadrons in minimum-bias p-p collisions at $\sqrt{s}=14 \mathrm{TeV}$, CMS Physics Analysis Summary CMS-PAS-QCD-08-004 (2008).

[16] PHOBOS Collaboration, B. B. Back et al., Charged particle pseudorapidity density distributions from Au+Au collisions at $\sqrt{s_{N} N}=130-G e V$, Phys. Rev. Lett. 87 (2001) 102303, [nucl-ex/0106006].

[17] CMS Collaboration, Study of Charged Hadron Multiplicity in Minimum Bias $p+p$ Collisions at $\sqrt{s}=900 \mathrm{GeV}$ and $10 \mathrm{TeV}$, CMS Physics Analysis Summary CMS-PAS-QCD-09-002 (2009).

[18] ALICE Collaboration, First proton-proton collisions at the LHC as observed with the ALICE detector: measurement of the charged particle pseudorapidity density at $\operatorname{sqrt}(\mathrm{s})=900 \mathrm{GeV}$, Eur. Phys. J. C65 (2010) 111-125, [arXiv:0911.5430].

[19] LHC Collaborations, "LHC report.” http://indico.cern.ch/conferenceDisplay.py?confId=76398, 2009. Web Page.

[20] CDF Collaboration, A. A. Affolder et al., Charged jet evolution and the underlying event in $p \bar{p}$ collisions at 1.8 TeV, Phys. Rev. D65 (2002) 092002.

[21] CDF Collaboration, D. E. Acosta et al., The underlying event in hard interactions at the Tevatron $\bar{p} p$ collider, Phys. Rev. D70 (2004) 072002, [hep-ex/ 0404004 ].

[22] CMS Collaboration, Measurement of the Underlying Event in Jet Topologies using Charged Particle and Momentum Densities, CMS Physics Analysis Summary CMS-PAS-QCD-07-003 (2007).

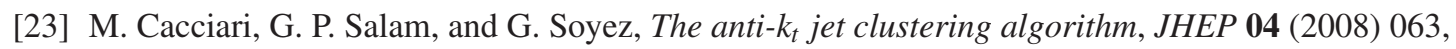
[arXiv:0802.1189].

[24] G. P. Salam and G. Soyez, A practical Seedless Infrared-Safe Cone jet algorithm, JHEP 05 (2007) 086, [arXiv:0704.0292].

[25] S. D. Ellis and D. E. Soper, Successive combination jet algorithm for hadron collisions, Phys. Rev. D48 (1993) 3160-3166, [hep-ph/9305266].

[26] S. Catani, Y. L. Dokshitzer, and B. R. Webber, The K-perpendicular clustering algorithm for jets in deep inelastic scattering and hadron collisions, Phys. Lett. B285 (1992) 291-299.

[27] S. Catani, Y. L. Dokshitzer, M. H. Seymour, and B. R. Webber, Longitudinally invariant K(t) clustering algorithms for hadron hadron collisions, Nucl. Phys. B406 (1993) 187-224.

[28] M. Wobisch and T. Wengler, Hadronization corrections to jet cross sections in deep-inelastic scattering, hep-ph/9907280.

[29] CDF Collaboration, T. Aaltonen et al., Measurement of the Inclusive Jet Cross Section at the Fermilab Tevatron p-pbar Collider Using a Cone-Based Jet Algorithm, Phys. Rev. D78 (2008) 052006, [arXiv:0807.2204].

[30] CDF - Run II Collaboration, A. Abulencia et al., Measurement of the Inclusive Jet Cross Section using the $k_{\mathrm{t}}$ algorithminp $\bar{p}$ Collisions at $\sqrt{s}=1.96 \mathrm{TeV}$ with the CDF II Detector, Phys. Rev. D75 (2007) 092006, [hep-ex/0701051].

[31] D0 Collaboration, V. M. Abazov et al., Measurement of the inclusive jet cross section in p $\bar{p}$ collisions at $\sqrt{s}=1.96 \mathrm{TeV}$, Phys. Rev. Lett. 101 (2008) 062001, [arXiv:0802.2400].

[32] CMS Collaboration, Initial Measurement of the Inclusive Jet Cross Section at 10 TeV with CMS, CMS Physics Analysis Summary CMS-PAS-QCD-08-001 (2009). 
[33] CMS Collaboration, Dijet Azimuthal Decorrelations in pp Collisions at $\sqrt{s}=10 \mathrm{TeV}$, CMS Physics Analysis Summary CMS-PAS-QCD-09-003 (2009).

[34] CMS Collaboration, Study of Hadronic Event-Shape Variables, CMS Physics Analysis Summary CMS-PAS-QCD-08-003 (2008).

[35] CMS Collaboration, CMS Search Plans and Sensitivity to New Physics using Dijets, CMS Physics Analysis Summary CMS-PAS-SBM-07-001 (2007).

[36] CMS Collaboration, Towards a measurement of the inclusive $W \rightarrow \mu \nu$ and $Z \rightarrow \mu^{+} \mu^{-}$cross sections in pp collisions at $\sqrt{s}=10 \mathrm{TeV}$, CMS Physics Analysis Summary CMS-PAS-EWK-09-001 (2009).

[37] CMS Collaboration, Towards a Measurement of the Inclusive $W \rightarrow e v$ and $\gamma^{*} / Z \rightarrow e^{+} e^{-}$Cross Sections in pp Collisions at $\sqrt{s}=10 \mathrm{TeV}$, CMS Physics Analysis Summary CMS-PAS-EWK-09-004 (2009).

[38] Particle Data Group Collaboration, C. Amsler et al., Review of particle physics, Phys. Lett. B667 (2008) 1.

[39] ATLAS Collaboration, N. Besson, M. Boonekamp, E. Klinkby, T. Petersen, and S. Mehlhase, Re-evaluation of the LHC potential for the measurement of Mw, Tech. Rep. SN-ATLAS-2008-070, 2008.

[40] ATLAS Collaboration, N. Besson, M. Boonekamp, E. Klinkby, T. Petersen, and S. Mehlhase, Re-evaluation of the LHC potential for the measurement of Mw, Eur. Phys. J. C57 (2008) 627-651, [arXiv:0805.2093].

[41] CMS Collaboration, Study of the $Z \rightarrow e^{+} e^{-}$differential cross section as a function of $Z$ rapidity at sqrts = 10TeV, CMS Physics Analysis Summary CMS-PAS-EWK-09-005 (2009).

[42] CMS Collaboration, Muon Differential Cross Section and Charge Asymmetry in Inclusive $p p \rightarrow W(\mu v)+X$ Production at $\sqrt{s}=10 \mathrm{TeV}$, CMS Physics Analysis Summary CMS-PAS-EWK-09-003 (2009).

[43] ATLAS Collaboration, “ATLAS Experiment - Public Results, Standard Model Further Results WZ Jets.” https://twiki.cern.ch/twiki/bin/view/Atlas/StandardModelFurtherResultsWZJets, 2008. Web Page.

[44] CMS Collaboration, Study of Z production in association with jets in proton-proton collisions at $\sqrt{s}=10 \mathrm{TeV}$ with the CMS detector at the CERN LHC, CMS Physics Analysis Summary CMS-PAS-EWK-08-006 (2009).

[45] CMS Collaboration, Prospects for the first measurement of the WW production cross-section in $p p$ collisions at $\sqrt{s}=10 \mathrm{TeV}$ center of mass energy with the CMS detector, CMS Physics Analysis Summary CMS-PAS-EWK-09-002 (2009). 